\title{
EFECTOS DE LA INCLUSIÓN FINANCIERA EN EL DESARROLLO HUMANO DEL DEPARTAMENTO DEL HUILA-COLOMBIA
}

\section{EFFECTS OF FINANCIAL INCLUSION ON HUMAN DEVELOPMENT OF THE DEPARTMENT OF HUILA-COLOMBIA}

\author{
Angy Katherine López-Puentes ${ }^{1}$, Luis Ángel Córdoba-Polania ${ }^{2}$ y Amanda Vargas-Prieto ${ }^{3}$ \\ ${ }^{1}$ Universidad de La Salle, Bogotá D.C, Colombia. Email: kathep96@gmail.com \\ ${ }^{2}$ Universidad de La Salle, Bogotá D.C, Colombia.Email: polania95@gmail.com \\ ${ }^{3}$ Universidad del Magdalena, Santa Marta, Colombia. Email: avargasp@unimagdalena.edu.co
}

Para citar este artículo: López, P. A., Córdoba, P. L. y Vargas, P. A. (2019). El rol de las cooperativas para la Inclusión Financiera en el desarrollo del departamento del Huila-Colombia. Clío América, 13(26), 340-349. doi:

http://dx.doi.org/10.21676/23897848.3576

Recibido: 20 junio de 2019

Aceptado: 11 de octubre de 2019

Publicado en línea: noviembre 28 de 2019

Palabras clave:

inclusión

financiera;

desarrollo

social; índice

de desarrollo

humano;

servicios

financieros;

cooperativas.

JEL: G21; G1; O20: G28.

\section{Keywords:}

financial

inclusion;

social

development

; HDI;

financial

services;

cooperatives.

\section{RESUMEN}

La inclusión financiera se ha convertido en un instrumento dentro de las políticas públicas que ejercen los países en busca de garantizar el acceso a servicios financieros. En Colombia se ha desarrollado el programa "Banca de las Oportunidades" con este propósito. Sin embargo, el programa no analiza el alcance de estos procesos en el bienestar de la población. En este artículo se analizan los efectos de la inclusión financiera en el desarrollo del departamento del Huila. En este sentido, se implementó una metodología mixta, mediante el método DEXPLIS; en la fase cuantitativa se estimó y analizó un modelo estadístico multivariado, a fin de determinar si existe relación entre inclusión financiera y desarrollo; en la fase cualitativa se aplicaron entrevistas a funcionarios de la Gobernación del Huila. Las técnicas de recolección de información fueron revisión de literatura, bases estadísticas y guía de entrevista. Dentro de los resultados obtenidos se determinó que existe una relación positiva entre el Índice de Desarrollo Humano (IDH) y los indicadores de inclusión financiera. Adicionalmente, se identificó que los indicadores de acceso y de uso repercuten en las dimensiones de salud, educación e ingreso siempre y cuando se garantice el uso de los productos y los servicios financieros.

\section{ABSTRACT}

Financial inclusion has become an instrument within public policies exercised by countries seeking change in access to financial services. In Colombia, the "Opportunity Banking" program has been developed for this purpose. However, it does not analyze the scope of these processes in the well-being of the population. This article analyzes the financial inclusion effects on the development of the department of Huila. In this regard, a mixed methodology was implemented, by using the DEXPLIS method. In the quantitative phase, a multivariate statistical model was estimated and analyzed to determine if there is a relationship between financial inclusion and development. In the qualitative phase, interviews were carried out with officials from the Huila Governorate. The information gathering techniques were the literature review, statistical bases and interview guide. Among the obtained results, it was determined that there is a positive relationship between the Human Development Index (HDI) and the indicators of financial inclusion. Besides, the indicators of access and use reflected in the health, education and income dimensions were identified as long as the use of financial products and services is guaranteed. 


\section{INTRODUCCIÓN}

Según el Banco Mundial (2018), la Inclusión Financiera $\neg($ IF) hace referencia al acceso a productos financieros que satisfagan las necesidades y a la vez sean útiles y asequibles para la población. De acuerdo con Alliance for Financial Inclusion (AFI), el análisis de la IF se mide a través de cuatro dimensiones: bancarización, uso, calidad e impacto sobre el bienestar social a partir de los servicios, y productos financieros (AFI, 2010).

El acceso al sistema financiero juega un papel crítico en la reducción de la pobreza y la promoción del crecimiento en todas las teorías del desarrollo (Adeola y Evans, 2017), de ahí que las economías de países de América Latina, como Brasil, Perú y Colombia, intensifiquen sus esfuerzos para elevar el grado de acceso a estos servicios dentro de sus políticas públicas, implementando estrategias e iniciativas que han permitido un mayor crecimiento y cubrimiento del sector financiero (Tafur, 2009).

A través de los estudios realizados (Sarma y Pais, 2011; Kuri y Laha, 2011; Nanda y Kaur, 2017; Zulaica, 2013; Unnikrishnan y Jagannathan, 2015; Kumar, Parimal y Bonnerjee, 2014; Adeola y Evans, 2017; Vargas y Yepes, 2018; Vargas y García, 2018; Dávila et al., 2018; Fajardo et al., 2018) se ha encontrado evidencia para indicar que existe una estrecha relación entre los procesos de IF (Nanda y Kaur, 2017; Kumar et al., 2014; Ortiz y Nuñez, 2017; Atkinson y Messy, 2013; Global Partnership for Financial Inclusion - GPFI, 2016; Banco Mundial, 2018; Grupo de Trabajo para la Medición de la Inclusión Financiera FIDWG, 2013; AFI, 2010; Asobancaria, 2015) y la mejora en las condiciones de vida, y que aquellos países con un alto nivel de IF presentan un mejor comportamiento del Desarrollo Humano (DH), analizado mediante alfabetización e ingresos (Williamson, 2015; Picazzo et al., 2011; Sen, 2000; Calderón, 2009; Ureña, 2014). El vínculo entre los procesos de IF y el nivel de desarrollo humano es de vital importancia, ya que asegurar el acceso a los servicios financieros se considera como un mecanismo efectivo para el alcance de una vida larga y saludable, mejores oportunidades para acceder al sistema educativo y reducir los niveles de pobreza: elementos asociados al IDH.

Para el caso colombiano, se destaca la estrategia liderada por el Gobierno nacional desde el 2006 con el programa Banca de las Oportunidades y que tiene como finalidad asegurar y proteger el acceso y uso a los servicios financieros, principalmente a personas con ingresos reducidos, con el propósito de incentivar el desarrollo económico, disminuir la pobreza e impulsar la inclusión social (Ministerio de Comercio, 2009). En cuanto a la dimensión de acceso, desde el 2015 se alcanzó cobertura total del sistema financiero, ya que el $100 \%$ de los municipios cuentan con presencia de alguna entidad financiera y las estadísticas de uso indican que el 80,1\% de la población posee al menos un producto financiero (Banca de las Oportunidades, 2017). Respecto a las dimensiones de calidad y bienestar, estas resultan mucho más complejas de determinar debido a que su cuantificación exige el desarrollo de estudios especializados no disponibles, razón por la cual no se desarrolla en los estudios realizados (Banca de las Oportunidades, 2011)

En lo que respecta al comportamiento del indicador de IF para el año 2016, en Colombia fue de 66,3 \%. Mientras tanto, la situación departamental para el mismo indicador muestra que el de Bogotá fue de 82,4\%; Casanare 78,9\%; Santander 73,8 \%; Risaralda 71,5 \% y Huila $70 \%$ (Banca de las Oportunidades, 2016). Respecto al departamento del Huila, a pesar de que presenta un indicador por encima del promedio nacional, aún la región requiere de una mayor profundización financiera (Asomicrofinanzas, 2018; Diario del Huila, 2018). De acuerdo con Asomicrofinanzas, el reto que se debe asumir es llegar con entidades crediticias a las regiones más apartadas y apoyar principalmente el sector agropecuario mediante el incentivo a otros bancos, para que tenga presencia en la región y se apoyen todos los negocios productivos del departamento.

Con relación a la zona de estudio, es importante mencionar que el Huila registra un crecimiento del PIB del 1,1\% (Banca de las oportunidades, 2016) lo que le permite contribuir a la economía colombiana con una participación de $1,8 \%$. Este departamento durante los últimos años ha avanzado en el tema de superación de pobreza, al pasar de un nivel de $58 \%$ en el 2008 a una incidencia de pobreza moderada de 44,3\%; al igual que reducir el nivel de desigualdad de 51,2 en el 2008 a 45,9 en 2016. Adicionalmente, presenta mejoría en términos de educación superior y aseguramiento a servicios de salud. De acuerdo con el Informe de Coyuntura Económica publicado por la Cámara de Comercio de Neiva (2017), estas características hacen de este departamento una zona de estudio interesante y de la cual no se ha realizado ninguno sobre la incidencia de la IF en el bienestar de sus pobladores.

A raíz de lo descrito anteriormente, se plantea como pregunta de investigación ¿cuáles son los efectos de la IF en el desarrollo del departamento del Huila? 
Se plantean tres objetivos específicos: analizar el comportamiento de los indicadores de inclusión financiera del departamento del Huila; caracterizar los indicadores de desarrollo del departamento del Huila e identificar la relación entre la inclusión financiera y el desarrollo del departamento del Huila.

\section{METODOLOGÍA}

Para efectos de la presente investigación se tiene en cuenta un método específico de investigación mixta denominado Diseño Explicativo Secuencial (DEXPLIS). Este método consta de dos fases: la primera recopila y analiza datos cuantitativos (datos expresados en forma numérica) y la segunda recopila y analiza datos cualitativos (datos expresados en propiedades no cuantificables).

\section{Fase cuantitativa}

La presente investigación es de alcance correlacional. Con respecto a la selección de variables, se analiza el grado de asociación entre variables de IF y DH. El periodo de análisis es desde agosto de 2008 a agosto de 2017, pues en este periodo se desarrolló el programa de Banca de las Oportunidades. Por facilidad en el acceso a la información, la periodicidad de los datos es bimestral. En cuanto a IF, se usa el número de corresponsales bancarios que representa la dimensión de acceso, y el número de cuentas de ahorro, en representación de la dimensión de uso. No obstante, la información recabada en torno a IF fue transformada, siguiendo la construcción de los indicadores del conjunto principal de IF propuesto por el grupo de trabajo para su medición (FIDWG), tal como se detalla en la tabla 1.

Tabla 1. Indicadores de IF

\begin{tabular}{|c|l|l|l|}
\hline Dimensión & \multicolumn{1}{|c|}{ Información inicial } & \multicolumn{1}{|c|}{ Nombre del indicador } & \multicolumn{1}{|c|}{ Fórmula utilizada } \\
\hline Acceso & $\begin{array}{l}\text { Número de } \\
\text { corresponsales } \\
\text { bancarios }\end{array}$ & $\begin{array}{l}\text { Número de puntos de acceso } \\
\text { por cada 10 000 habitantes }\end{array}$ & $\begin{array}{l}\text { (Número total de puntos de } \\
\text { acceso/ población adulta } \\
\left.\text { total }{ }^{1}\right)^{*} 10000\end{array}$ \\
\hline Uso & $\begin{array}{l}\text { Número de Cuentas } \\
\text { de ahorro }\end{array}$ & $\begin{array}{l}\text { Número total de cuentas de } \\
\text { depósito reguladas por cada } \\
10000 \text { adultos }\end{array}$ & $\begin{array}{l}\text { (Número total de cuentas de } \\
\text { depósito reguladas/ población } \\
\text { adulta total)* 10 000 }\end{array}$ \\
\hline
\end{tabular}

Fuente: elaboración propia basado en AFI (2010).

La información revisada fue el número de corresponsales bancarios y número de cuentas de ahorro, información utilizada para la construcción de los indicadores del conjunto principal. Las cifras del Indice de Desarrollo Humano (IDH) se calculan en periodicidad anual; por lo tanto, fue necesario desagregar la serie ajustándola a través de la metodología de Eviews y convertir los datos anuales en datos bimestrales.

\section{Método estadístico multivariado}

Con la finalidad de evaluar los efectos de la IF en el DH se realizó un método estadístico multivariado a través de una regresión múltiple. Dicho modelo permitió evaluar el efecto de dos o más variables explicativas o independientes $\left(\mathrm{X}_{2}\right.$ y $\left.\mathrm{X}_{3}\right)$, sobre una variable dependiente $(\mathrm{Y})$ (Gujarati y Porter, 2010). La notación del modelo de regresión múltiple se expresa a través de la Función de Regresión Poblacional (FRP), tal como se muestra a continuación:

$$
Y_{i}=\beta_{1}+\beta_{2} X_{2 i}+\beta_{3} X_{3 i}+u_{i}
$$

Donde $\mathrm{Y}$ es la variable dependiente, $\mathrm{X}_{2}$ y $\mathrm{X}_{3}$ las variables explicativas, u es el término de perturbación estocástica, $\mathrm{i}$ la i-ésima observación y los coeficientes $\beta 2$ y $\beta 3$ son coeficientes de regresión parcial.

Para efectos de la presente investigación se tiene que la variable dependiente es el IDH y las variables explicativas son el número de puntos de acceso por cada 10000 habitantes y el número total de cuentas de depósito reguladas por cada 10000 adultos. Siendo así, la notación del modelo de regresión múltiple se expresa tal como se observa en la siguiente ecuación:

$\mathrm{IDH}=\beta_{1}+\beta_{2}$ Número de

puntos de acceso por cada 10

000 habitantes $+\beta_{3}$ Número

total de cuentas de depósito

reguladas por cada 10000

$$
\text { adultos }+\mathrm{u}
$$

\footnotetext{
${ }^{1}$ Para efectos de la investigación, la población adulta es aquella mayor a los 15 años de edad o más.
} 
Para el análisis cuantitativo de los datos se recurrió al uso del programa de análisis estadístico EViews $10^{2}$, que es un software econométrico que ofrece el acceso a herramientas estadísticas, de pronóstico y modelado. El modelo econométrico estimado fue el siguiente:

$$
\mathrm{IDH}=0,678129+0,000586
$$

Número de puntos de acceso por cada 10000 habitantes + 0,000000111 Número total de cuentas de depósito reguladas por cada 10000 adultos

Adicionalmente, con la finalidad de determinar el efecto de la IF en cada una de las dimensiones del DH, se estimaron tres modelos adicionales, en los que la variable dependiente corresponde a cada componente del IDH.

Si se tiene como variable dependiente Longevidad, el modelo estimado es el siguiente:

$$
\begin{gathered}
\text { Longevidad (Salud) }=0,790047 \\
+0,001411 \text { Número de puntos } \\
\text { de acceso por cada } 10000 \\
\text { habitantes }+0,000000132 \\
\text { Número total de cuentas de } \\
\text { depósito reguladas por cada } 10 \\
\text { 000 adultos }
\end{gathered}
$$

Si se tiene como variable dependiente Alfabetización, el modelo estimado es el siguiente:

$$
\begin{gathered}
\text { Alfabetización (Educación) }= \\
0,568233-0,000290 \text { Número } \\
\text { de puntos de acceso por cada } 10 \\
000 \text { habitantes }+0,0000000998 \\
\text { Número total de cuentas de } \\
\text { depósito reguladas por cada } 10 \\
000 \text { adultos }
\end{gathered}
$$

Si se tiene como variable dependiente Niveles decentes de vida, el modelo estimado es el siguiente:

$$
\begin{gathered}
\text { Niveles decentes de vida } \\
\text { (Ingreso) }=0,697810+ \\
0,001032 \text { Número de puntos de } \\
\text { acceso por cada } 10000 \\
\text { habitantes }+0,0000000648 \\
\text { Número total de cuentas de } \\
\text { depósito reguladas por cada } 10
\end{gathered}
$$

\section{0 adultos}

Con la finalidad de verificar la validación del modelo multivariado y la significancia individual de cada uno de los parámetros se realiza una prueba de hipótesis estadística analizando la probabilidad. Esta prueba de significancia tuvo como hipótesis nula (Ho) que los coeficientes son cero, y como hipótesis alternativa (Ha) que los coeficientes son diferentes de cero; si la probabilidad es menor al alfa del $5 \%(0,05)$ se rechaza la hipótesis nula, por lo cual los coeficientes son significativos para el modelo (Gujarati y Porter, 2010).

En cuanto al modelo que determina la relación entre la IF y el IDH, se revisó la significancia individual de los coeficientes (indicador de acceso e indicador de uso). Esto se hizo a través de las probabilidades dadas en la salida del modelo corrido en Eviews 10, en donde se buscó que dichas probabilidades tuvieran un valor menor al alfa del $5 \%$ $(0,05)$. Como resultado, ambos indicadores arrojaron una probabilidad menor al $5 \%$, lo que significa que las variables independientes son significativas e ideales para explicar a la variable dependiente IDH y explican en buena medida el modelo

El valor de $\mathrm{R}^{2}$ se encuentra entre 0 y 1 ; el ajuste del modelo es “mejor" entre más cerca esté $\mathrm{R}^{2}$ a 1 . Esta medida es de gran importancia, puesto que se constituye como una medida global del grado en que la variación en una variable determina la variación en la otra (Gujarati y Porter, 2010). Para efectos de la presente investigación, una vez se ejecutó el programa, los resulados arrojados de $\mathrm{R}^{2}$ correspondieron a un valor de 0,842820 , lo cual indica que el 84,2820 \% de la variación en el IDH se explica mediante el número de puntos de acceso por cada 10000 habitantes y el número total de cuentas de depósito reguladas por cada 10000 adultos.

\section{Fase cualitativa}

Con la finalidad de analizar los datos recabados en la fase cuantitativa se aplicaron las entrevistas a funcionarios de la Gobernación del Huila y al Instituto Financiero para el Desarrollo del Huila - INFIHUILA. Inicialmente, se realizaron cuatro entrevistas en la sala de juntas de INFIHUILA, en la ciudad de Neiva, Huila; la fecha de realización de las entrevistas corresponde al jueves 11 de abril del año 2019. Dicha entrevista contó con la presencia de tres directivos de INFIHUILA y un delegado de la Gobernación del Huila. Posteriormente, se realizó una entrevista en el Departamento Administrativo de 
Angy Katherine López-Puentes, Luis Ángel Córdoba-Polania y Amanda Vargas-Prieto

Planeación, el jueves 11 de abril de 2019 en la ciudad de Neiva.

\section{RESULTADOS}

En este apartado se encuentran los principales efectos de la

IF en el desarrollo humano. En primer lugar, se presenta el resultado de la matriz de correlación. Luego, se presentan los efectos del indicador de acceso y el indicador de uso en el desarrollo humano, y finalmente se analizan los efectos de la IF sobre cada uno de los componentes del IDH.

\section{Resultados de la matriz de correlación}

En cuanto a la matriz de correlación de la tabla 2, esta indica que sí existe una relación lineal directa (r: 1), no hay relación lineal (r: 0) o hay relación inversa (r: -1) entre las variables explicativas (Gujarati y Porter, 2010).

Tabla 1. Matriz de Correlación

\begin{tabular}{|l|r|r|r|}
\hline Matriz de Correlación & \multicolumn{1}{|l|}{ IDH } & Indicador de Acceso & \multicolumn{1}{|c|}{ Indicador de Uso } \\
\hline IDH & 1,000000 & 0,908085 & 0,884140 \\
\hline Indicador de Acceso & 0,908085 & 1,000000 & 0,913033 \\
\hline Indicador de Uso & 0,884140 & 0,913033 & 1,000000 \\
\hline
\end{tabular}

Fuente: elaboración propia basada en datos obtenidos en Eviews10.

En la tabla 2 se observa el resultado de la matriz de correlación para el modelo en el que se tiene como variable dependiente el IDH, y como variables explicativas los indicadores de IF. Dicho resultado indica que hay una relación directa entre el IDH y el indicador de acceso, y el IDH y el indicador de uso. Por último, el indicador de acceso y el indicador de uso también tienen relación.

\section{Efecto del Acceso al Sistema Financiero en el DH para el Departamento del Huila}

A través de la matriz de correlación se determina que existe una relación directa entre el indicador de acceso, el cual corresponde a número de puntos de acceso por cada 10000 habitantes, y la variable del IDH. De igual forma, este indicador tiene un mayor efecto dentro de la variable IDH que el indicador de uso. Sin embargo, dicho efecto no es significativo, a raíz de que un cambio en el indicador de acceso no genera cambios sustanciales en la variable IDH ya que cuando el indicador de acceso varía en una unidad, el IDH varía en un mismo sentido 0,000586 .

Por otro lado, se identificó que tener acceso a los productos y los servicios financieros mejora la calidad de vida de las personas. Desde la perspectiva de Osorio, a nivel departamental existen fondos y recursos que apoyan a las empresas de familia y microempresas, buscando facilitar el acceso a los recursos para que las personas puedan sostenerse y sobrevivir empresarialmente; es ahí donde se refleja una mejora e impacto (Osorio, Comunicación personal, 11 de abril de 2019).

Adicionalmente, tener acceso al sistema financiero facilita tener oportunidades en educación, vivienda y desarrollo del ser humano. Figueroa señala que tener acceso a servicios y productos financieros es el "puente entre los sueños que tiene una persona y la realización de estos, permite el acceso a vivienda, educación (...) hace parte y permite el desarrollo de cada individuo" (Figueroa, Comunicación personal, 11 de abril de 2019). Igualmente, se menciona que para el gobierno departamental es de suma importancia apoyar los emprendimientos como herramienta de oportunidad para los habitantes, para que a través de esto puedan obtener mejoras en su bienestar (Herrera, Comunicación personal, 11 de abril de 2019).

Durante el periodo analizado es evidente el aumento exponencial de los puntos de acceso en el departamento del Huila, y este avance ha estado estrechamente relacionado con la adopción de nuevas tecnologías en el sistema financiero y el desarrollo urbanístico que ha experimentado la región; lo anterior ha permitido que el sistema financiero tenga presencia y cobertura en zonas apartadas del casco urbano y que un mayor número de población tenga al alcance estas herramientas, servicios y productos que ofrece el sistema financiero.

En gran medida, el avance en materia de acceso al sistema financiero es explicado por la gran participación del sector solidario en la región, el cual se interesa por ejecutar su labor en pro de la población rural, vulnerable y de bajos ingresos. En ese caso, las cooperativas financieras se han convertido en un modelo eficiente para la IF, porque al "sistema financiero tradicional no le interesa esta población (...) a los bancos no les interesa hacer convenios para atender a las mypimes ni mucho menos las famiempresas, entonces se ha tenido que acudir a las cooperativas" (F, 
Osorio, Comunicación personal, 11 de abril de 2019).

De esta manera, el departamento del Huila trabaja con las cooperativas financieras realizando alianzas estratégicas. A modo de ilustración se encuentran los proyectos con la Secretaría de Agricultura, que buscan implementar fondos para la mujer productiva y con los que mujeres rurales pueden acceder a recursos" (Yepes, Comunicación personal, 11 de abril de 2019).

Asimismo, los proyectos que lidera la gobernación a través del INFIHUILA en pro de la población, crean vínculos con las cooperativas para que cumplan el papel de operadores financieros, depositando los recursos directamente a las personas. Este tipo de alianzas se convierten en mecanismos para garantizar la IF pues se trabaja para que cualquier persona pueda acceder a dichos recursos (Figueroa, Comunicación personal, 11 de abril de 2019).

Por consiguiente, el acceso al sistema financiero ha aumentado a través de la complementariedad con las cooperativas del departamento al eliminar barreras que impiden aprobar recursos. Según Figueroa, se han diseñado estrategias para que las personas que lleguen con una idea de negocio, sin importar su estrato, puedan tener la oportunidad de acceder a los recursos.

\section{Efecto del Indicador de Uso sobre el DH en el Departamento del Huila}

Inicialmente, se identificó que el indicador de uso tiene un efecto casi nulo en el IDH puesto que cuando el indicador de uso varía en una unidad, el IDH varía en un mismo sentido 0,000000111. No obstante, al analizar la matriz de correlación, es notorio que existe una relación positiva entre las variables IDH e indicador de uso.

Adicionalmente, se determina que para mejorar las condiciones de vida no es suficiente con tan solo acceder a productos y servicios financieros, pues para esto es necesario el aprovechamiento efectivo de los productos y los servicios a los cuales se pueda acceder. Para Osorio "acceder como tal no genera ningún cambio, está el producto o servicio, pero no genera cambio, lo importante es usar el sistema" (Osorio, Comunicación personal, 11 de abril de 2019). Esta perspectiva es apoyada desde la posición de Figueroa quien señala que "para garantizar mejores condiciones de vida la población necesariamente debe poder acceder, pero igual usar los productos a los que acceda" (Figueroa, Comunicación personal, 11 de abril de 2019).

En suma, se evidencia que el indicador de uso no es relevante y significativo para explicar los efectos dentro del DH (IDH). Esto puede ser ocasionado por la medida utilizada, número de cuentas de ahorro, puesto que generalmente el sistema financiero autoriza la apertura de cuentas de ahorro a otro tipo de productos que ellos mismos ofrecen, lo que se traduce como abandono de las cuentas y no garantiza el uso de las mismas.

\section{Efecto de la IF en Salud de los habitantes del Huila}

Hay que tener en cuenta, que para medir el DH se utiliza la longevidad a través de la esperanza de vida, y que mediante este componente se pueden interpretar buenas condiciones de alimentación y salud de la población. Por lo tanto, la variable dependiente es longevidad y las variables explicativas corresponden a número de puntos de acceso por cada 10000 habitantes (indicador de acceso) y número total de cuentas de depósito reguladas por cada 10000 adultos (indicador de uso). Una vez ejecutado el programa se obtiene un valor de $\mathrm{R}^{2}$ de 0,899541 , lo cual indica que el $89,9541 \%$ de la variación en longevidad se explica mediante el indicador de acceso y el indicador de uso. Además, se puede determinar que el indicador de acceso sobre longevidad es poco representativo, puesto que cuando este varía en una unidad, la variable dependiente presenta un cambio de 0,001411 . En cuanto al indicador de uso se puede mencionar que este no explica la variable longevidad, ya que no es significativo en el modelo.

La IF tiene un efecto favorable dentro de la salud, pues repercute en la calidad de vida de las personas. Una vez la población accede y usa los productos financieros, puede distribuir sus ingresos para obtener mejores servicios. De acuerdo con Figueroa, la IF trae DH pues las personas pueden prosperar con sus ideas de negocio y proyectos de vida; y de la mano de esto debe necesariamente venir la posibilidad de poder acceder al servicio de salud; no a través del sistema de salud tradicional sino, al haberse desarrollado económicamente, acceder a una salud privilegiada (Figueroa, Comunicación personal, 11 de abril de 2019).

\section{Efecto de la IF en la Educación de la Población del Huila}

Tal como se mencionó en el marco teórico, para analizar los niveles de DH es esencial identificar la situación de la población en términos de alfabetización. De esta manera, al determinar el efecto de la IF en la educación de los habitantes del Huila, se encontró que los indicadores de IF no explican la variable alfabetización. Esto sucede a raíz de que las variables no son significativas en el modelo estimado. Además, el modelo se explica muy poco, puesto que el valor de $\mathrm{R}^{2}$ es de 0,056323 , lo que indica que el 
Angy Katherine López-Puentes, Luis Ángel Córdoba-Polania y Amanda Vargas-Prieto

grado de asociación de las variables es débil. Sin embargo, desde la perspectiva cualitativa es evidente que la IF tiene un impacto dentro de la mejora del nivel educativo de la población.

Es importante mencionar que, al elevarse las oportunidades para acceder y usar los productos y servicios financieros, se permite ingresar a educación de mejor calidad. Como lo expresa Figueroa desde su experiencia personal, los productos y servicios financieros le permitieron culminar sus estudios universitarios (Figueroa, Comunicación personal, 11 de abril de 2019). Adicionalmente, Yepes menciona que el Gobierno departamental, a través del Fondo Genaro Díaz Jordán, ha impulsado la IF enfocada en la educación de la población menos favorecida, lo que ha permitido que muchas personas accedan a través de créditos condonables a la educación superior (Yepes, Comunicación personal, 11 de abril de 2019).

En la actualidad, el Gobierno del departamento del Huila tiene la educación como eje central de su plan de desarrollo. Esto se puede evidenciar desde su lema "el camino es la educación". De esa manera, desde el gabinete gubernamental se han liderado proyectos en conjunto con el sector solidario, para que la mayoría de la población acceda a productos y servicios financieros que le permitan vincularse al sistema educativo y obtener educación de alta calidad (Herrera, Comunicación personal, 11 de abril de 2019).

Como resultado, desde el análisis cuantitativo la relación entre las variables de IF y educación no existe. No obstante, la perspectiva cualitativa señala que la relación entre dichas variables es evidente a nivel departamental, ya que la IF ha facilitado a los habitantes el acceso a servicios de educación de calidad. A modo de ilustración, el departamento del Huila mejoró en cobertura bruta (cantidad o porcentaje de la totalidad de estudiantes matriculados en el sistema educativo) en formación universitaria, puesto que alcanzó un porcentaje de 4,10\% estudiantes matriculados en el sistema educativo superior en 2017, después de presentar niveles bajos de 2,82 \% (Cámara de Comercio de Neiva, 2017). Por lo tanto, se deduce que cada vez un número mayor de estudiantes está accediendo a servicios educativos.

\section{Efecto de la IF en el Ingreso de los Habitantes del Huila}

En lo que respecta al análisis de los niveles decentes de vida, componente del IDH, para su medición se recurre al PIB per cápita. Así, se identifican los efectos de la IF en el ingreso donde la variable dependiente es Niveles Decentes de Vida y las variables explicativas corresponden a indicador de acceso e indicador de uso. Para este modelo estimado se encuentra que el valor de $\mathrm{R}^{2}$ es de 0,868153 ; lo cual indica que el $86,8153 \%$ de la variación en los niveles decentes de vida se explica mediante mencionadas variables. Aunque el ajuste del modelo es adecuado, ya que el $\mathrm{R}^{2}$ es cercano a 1 , se evidencia que el indicador de uso no es significativo para este modelo.

Asimismo, se establece que IF tiene un efecto positivo sobre el ingreso de las personas. Desde la perspectiva de Osorio, la IF, más allá de aumentar el ingreso, genera oportunidades con las cuales las personas podrían llegar a aumentarlo (Osorio, Comunicación personal, 11 de abril de 2019). En contraste, Figueroa respalda esta posición, siempre y cuando las personas que cuenten con estas oportunidades tengan claridad del uso que se le va a dar a los recursos de los cuales son beneficiarios, lo que está relacionado con educación financiera (Figueroa, Comunicación personal, 11 de abril de 2019). Adicionalmente, Herrera señala que las oportunidades que otorga la IF a las personas permite que estas puedan proyectarse a nivel personal, familiar, profesional y empresarial (Herrera, Comunicación personal, 11 de abril de 2019).

\section{DISCUSIÓN}

Para evidenciar verdaderos efectos de la IF en el desarrollo de la población del departamento del Huila, el reto que se debe asumir es avalar el acceso y el uso de los servicios y los productos financieros; esto para aprovechar todos los beneficios que aporta un proceso de IF. Por tanto, a nivel departamental se deben gestionar mecanismos para que los individuos que accedan al sistema financiero adquieran un producto o servicio, permanezcan activos con esos productos y reciban el bienestar a través de la utilización eficiente y segura. Igualmente, las entidades financieras deben lograr ofrecer productos de ahorro innovadores e incentivos para que los consumidores financieros optimicen el uso de los productos.

La investigación estuvo limitada por la disponibilidad de la información. En primer lugar, la construcción tanto del indicador de acceso y uso estuvo condicionada a la falta de publicación de todas las variables que analizan puntos de acceso, como número de datáfonos y número de oficinas; y de uso, como el análisis del comportamiento de tarjetas de crédito y débito, composición de crédito de viviendas, entre otros. En segundo lugar, los datos de IDH se encuentran limitados al cálculo de Global Data Lab, un ente internacional, pues a nivel departamental no existe el cálculo de este índice, y la información para calcularlo tampoco está a la mano del público. Por esto, es recomendable que se sigan realizando estudios sobre el 
tema y que el uso de los indicadores se realice a nivel departamental y no solo nacional.

\section{CONCLUSION}

Esta investigación se desarrolló con el propósito de identificar los efectos de la IF en el desarrollo del departamento del Huila, a través del análisis del comportamiento de los indicadores de IF y la caracterización del IDH; y, por último, determinar la relación entre ambos índices en dicha región. De esta manera, se estableció que asegurar el acceso a los servicios financieros, se considera como una herramienta útil para promover el desarrollo humano de la población. Además, los niveles de desarrollo humano pueden ser mejorados a través de la IF.

Adicionalmente, se logró determinar que existe una relación positiva entre los indicadores de IF y el desarrollo humano medido a través del IDH en el departamento del Huila. Teniendo en cuenta lo anterior, se encontró que el comportamiento de los indicadores de IF en el departamento son ascendentes, puesto que la región ha experimentado un aumento de 1 punto de acceso a 26 por cada 10000 personas, al igual de 6794 a 17645 cuentas de depósito por cada 10000 habitantes, lo que representa un avance en términos de IF a nivel departamental. En lo que respecta al IDH del departamento se identificó un leve comportamiento ascendente.

\section{Declaración sobre conflictos de interés}

Los autores de este documento afirman que son independientes con respecto a las instituciones financieras y de apoyo. Además, los autores afirman que durante la realización de la investigación o la redacción del manuscrito, no ha habido intereses o valores diferentes de los que habitualmente sigue una investigación científica.

\section{REFERENCIAS BIBLIOGRÁFICAS}

Adeola, O. y Evans, O. (2017). Financial inclusion, financial development, and economic diversification in Nigeria. Journal of Developing Areas, 51(3), 115.

Alliance for Financial Inclusion - AFI. (2010). Documento sobre políticas, la medición de la IF para entes reguladores: Diseño e implemetación de encuestas. AFI. https://www.afi- global.org/sites/default/files/pdfimages/ afi\%20policy\%20paper-SP\%20(2).pdf

Asobancaria. (2015). El camino hacia la inclusión financiera. https://www.asobancaria.com/sabermass ermas/wpcontent/uploads/2015/fasciculos/la_banc a_mas_cerca_de_usted/FASCICULO_4 .pdf

Asomicrofinanzas. (2018). Asociación colombiana de instituciones microfinancieras. http://www.asomicrofinanzas.com.co/i mages/publicaciones/notas\%20de $\% 20$ microfinanzas\%20-\%20agosto.pdf

Atkinson, A, \& Messy, F. (2013). Promoting Financial Inclusion through Financial Education: OECD/INFE Evidence, Polices and Practice.

Banca de las Oportunidades. (2011). Reporte de IF. (Serie de publicaciones sobre Inclusión Financiera). Banca de las oportunidades.

http://bancadelasoportunidades.gov.co/s ites/default/files/201802/PDF\%202011_0.pdf

Banca de las Oportunidades. (2016). Reporte de IF. (Serie de publicaciones sobre Inclusión Financiera). Banca de las oportunidades.

https://bancadelasoportunidades.gov.co/ index.php/en/reportes/314

Banca de las Oportunidades. (2017). Reporte de IF. (Serie de publicaciones sobre Inclusión Financiera). Banca de las oportunidades. https://bancadelasoportunidades.gov.co/ index.php/en/reportes/314

Banco Mundial. (2018). La IF es un factor clave para reducir la pobreza e impulsar la prosperidad.

https://www.bancomundial.org/es/topic/ financialinclusion/overview 
Calderón, R. (2009). Ciencia, desarrollo humano y Calidad de vida. Hallazgos, Revista de investigaciones, 6(12), 47-57.

Cámara de Comercio de Neiva. (2017). Informe de Coyuntura Económica. https://ccneiva.org/serviciosempresariales/informes-economicos/

Dávila, R., Vargas, A., Blanco, L., Roa, E., Cáceres, L.E., Vargas, L.A. (2018): "Características de la economía solidaria colombiana. Aproximaciones a las corrientes influyentes en Colombia", CIRIEC- España, Revista de Economía Pública, Social y Cooperativa, 93, 85113, DOI: 10.7203/CIRIEC-E.93.10327. https://ojs.uv.es/index.php/ciriecespana/ article/view/10327

Diaro del Huila. (2018). Huila requiere mayor profundización financiera. https://diariodelhuila.com/huilarequiere-mayor-profundizacionfinanciera

Fajardo, K.N., Vargas Prieto, A. (2018). Reconocimiento social en el marco del posconflicto en Colombia: el caso de las zonas de reserva campesina. Revista Cooperativismo y desarrollo. ISSN: 0120-7180 ed: Universidad Cooperativa de Colombia, Vol. 26 No. 112, edición enero de 2018. https://revistas.ucc.edu.co/index.php/co/ article/view/2189

Global Partnership for Financial InclusionGPFI. (2016). Financial Inclusion Indicators-

G20.http://www.gpfi.org/sites/default/fil es/G20\%20Set\%20of\%20Financial\%20 Inclusion\%20Indicators.pdf

Grupo de Trabajo para la Medición de la Inclusión Financiera-FIDWG. (2013).

y libertad universal. Estudios sociales, 19(37), 253-279.

http://www.scielo.org.mx/scielo.php?scr ipt=sci_arttext \&pid=S0188-

$45572011000100010 \& \operatorname{lng}=e s \& t \operatorname{lng}=e s$.
Medición de la Inclusión FinancieraConjunto Principal de Indicadores de Inclusión Financiera. Alliance for Financial Inclusion. https://www.gob.mx/cms/uploads/attach ment/file/69640/Indicadores_AFI.pdf

Gujarati, D. y Porter, D. (2010). Econometría. México: McGraw-Hill.

Kumar, S., Parimal, S. J. y Bonnerjee, S. (2014). Impact of Banking Penetration on Economic Growth: A State-wise Comparative Study. Business Perspectives \& Research, 2(2), 47-64

Kuri, P. K. y Laha, A. (2011). Financial Inclusion and Human Development in India: An Inter-State Analysis. Indian. Journal Of Human Development, 5(1), 61.

Ministerio de Comercio. (2009). Qué es la Banca de las Oportunidades? http://www.mipymes.gov.co/financiacio n-para-el-desarrollo-empresarial/lineasde-credito/microfinanzas/como-sepuede-acceder-a-ellas/banca-deoportunidades

Nanda, K. y Kaur, M. (2017). Bank-led Financial Inclusion and Human Development: Evidence from India. South Asian Journal Of Management, 24(1), 114-140.

Ortiz Montes, S., \& Núñez Tabales, J. (2017). Inclusión Financiera: Diagnóstico de la situación en América Latina y el Caribe. Revista Galega de Economía, 26 (1), 45-54.

Picazzo, E., Gutiérrez, E., Infante, J., \& Cantú, P. (2011). La teoría del desarrollo humano y sustetable: hacia el reforzamiento de la salud como derecho

Sarma, M. y Pais, J. (2011). Financial Inclusion and Development. Journal of International Development, 23(5), 613-628. 
Sen, A. (2000). Desarrollo y Libertad. Buenos Aires: Editorial Planeta. https://www.palermo.edu/Archivos_con tent/2015/derecho/pobreza_multidimens ional/bibliografia/Sesion1_doc1.pdf

Tafur, C. (2009). Bancarización: Una aproximación al caso colombiano a la luz de América Latina. Estudios Gerenciales, 25(1), 13-37.

Unnikrishnan, R. Jagannathan, L. (2015). Unearthing Global Financial Inclusion levels and analysis of financial inclusion as a mediating factor in global human development. Serbian Journal of Management, 10(1), 19-32.

Ureña Villamizar, Y.C. (2014). Cultura Innovativa: Prospectiva de las condiciones estratégicas para el desarrollo del capital humano. Clio America, 8(16), 195-200.

Vargas Prieto, A., García Estévez, J. (2018). “El impacto del sector minero energético en el desarrollo social de las regiones: en caso del Catatumbo, Colombia" en Estudios sobre medio ambiente y sostenibilidad: una mirada desde Colombia/Hugo Fernando Guerrero Sierra y otros. Bogotá, Universidad Santo Tomás Seccional Tunja. 490 p. ISBN 978-958-5471-12-2, pp. 193-216.

Vargas Prieto, A., Yepes Lugo, C. (2018). Aportes teóricos y metodológicos sobre el emprendimiento agrícola. En: Desarrollo, estructuras económicas, políticas públicas y gestión. Reflexión interdisciplinar. Ciudad de México, México: De la Salle Ediciones. ISBN: 978-958-5486-23-2.

Williamson, N. (2015). Análisis del cálculo del índice de desarrollo humao municipal de Nicaragua. Revista Cientifica Electrónnica de Ciencias Humanas, 11(31), 58-78
Zulaica, P. C. (2013). Financial Inclusion Index: Proposal of a Multidimensional Measure for México. Revista Mexicana de Economía y Finanzas. Nueva Época / Mexican Journal of Economics and Finance, 8(2), 157-180. 